\title{
Major Pharmacy Practice Challenge for India: Irrational Fixed Dose Combinations
}

\author{
Kunjal Patel, Prachie Patel, M. Chandra Sekar \\ College of Pharmacy, University of Findlay, UNITED STATES.
}

\begin{abstract}
During the advanced pharmacy practice rotation in India in the winter of 2017, The University of Findlay Pharmacy students observed the availability and sale of large number of fixed dose combinations and many of those combinations made no pharmacological sense. While availability of "irrational combinations" in Indian market is old news, what is new in this report is our observation that unless pharmacy practitioners in India are able to eliminate these proliferation of irrational combinations from the market-it will suffocate the growth of the nascent field of pharmacy practice that is being promoted by awarding doctor of pharmacy degree at the Indian educational institutions.

Key words: Fixed dose combination, Pharmacy Practice, India,Clinical Pharmacy, Barrier, Pharm D.
\end{abstract}

Fixed dose combination (FDC) consists of two or more active ingredients in a single pill that are preferred when patient requires multiple regimens to control particular disease state i.e. various antihypertensive agents to control blood pressure or antidiabetics to control blood sugar and A1C. These combination products make patients life simpler by reducing pill burden. For FDC products to be on the market, it must meet specific requirements to be accepted. ${ }^{1}$ Some things that need to be considered when creating FDC products include- 1) active ingredients in the combination should have different mechanisms of actions, 2) pharmacokinetics should differ, and 3) combining such drugs should not cause excessive toxicity. ${ }^{1}$

Fixed dose combinations (FDCs) are quite beneficial for patients who need to take more than one medication to treat one or more conditions. Using FDC products reduces pill burden, especially for patients who tend to forget to take a dose or those who are merely non-compliant. ${ }^{2}$ The more pills a patient has to take, there is a chance of treatment failure, relapse, and the possibility of drug resistance with single agents. ${ }^{2}$ FDCs can lead to better outcome of case management in such cases. ${ }^{2}$ These include greater patient compliance, easier dosing schedule, lower risk for side effects, and lower cost. ${ }^{2}$ Some FDCs may also produce a synergistic effect, an additional positive benefit. ${ }^{2}$

While appropriate use of FDCs has advantages, inappropriate use may be accompanied by serious downsides to these types of products. When creating a single pill of two or more medications, one key thing to look out for is drug interactions. ${ }^{2}$ Drug interactions can happen between active and inactive ingredients in the pill, which can lead to chemical instability. ${ }^{3}$ Another disadvantage with FDCs is the inability to change dosing. ${ }^{2}$ FDC products have a fixed dose of various active drug in every single pill, therefore if a higher or lower dose is needed to treat a particular condition, it may not be easily achievable. $^{2}$

While FDCs are available in the United States, there are many more of them available in the Indian pharmaceutical market. ${ }^{1}$ There are over 6000 FDC products
DOI: 10.5530/ijopp.11.2.13

Address for correspondence: Prof. M. Chandra Shekar, College of Pharmacy, Univesity of Findlay, UNITED STATES.

Phone no: (001) 5132588747 Email Id: sekar@findlay.edu

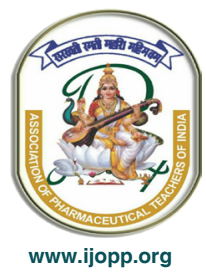


in the Indian market; and many of those combination can be considered "irrational", as there is no scientific rational for such combinations; use of such "irrational combinations" can only lead to harm ${ }^{1-2}$ International drug regulators are raising questions about the quality of FDC products manufactured in India. ${ }^{3}$ There has been an issue of manufacturers creating these products that very often include drugs from the same therapeutic class that can place the patient at an increased risk of adverse events. ${ }^{3}$ Other concerns include laws and regulations regarding marketing and availability of FDC products. The Central Drugs Standard Control Organization (CDSCO) is the national regulator of Indian pharmaceuticals that banned sales of around 294 FDC products back in 2007 due to the fact that these combination products were not approved by the central regulator. ${ }^{3}$ Many FDC products are manufactured by Indian firms without approval by CDSCO. However, they are still able to receive manufacturing/distribution licenses to be on the market following approval by state agencies, ${ }^{3-4}$ due to a large loophole in the law. Overall, disregard for rules and regulations by the public and law enforcement resulted in CDSCO not being taken seriously. ${ }^{3}$ For example, a study was conducted in India between 2011-2012. Out of 124 NSAID-FDC products available in the market, only 34 were officially approved by $\mathrm{CDSCO} ;{ }^{4}$ Out of 25 metformin FDC products available, only 20 were approved by CDSCO; ${ }^{4}$ and of 16 antidepressant and benzodiazepine FDCs in the market - only 3 were approved by CDSCO. ${ }^{4}$ These findings are in clear contrast to UK and USA where only approved FDCs are available in those countries. ${ }^{4}$ The number of combinations have continued to raise over the years - previously 124 NSAID combinations have now become 2,739; now available metformin products number 536, and antidepressant/ benzodiazepine number 211 products. ${ }^{3}$ Many of the unapproved FDC formulations may not have been tested for safety or efficacy before being on the market, which can lead to serious adverse events to patients exposed to these products. ${ }^{3}$ One primary reason for such proliferation of FDCs in India is manufacturers are able to override the price control established by government for single ingredient products.

India has a wide variety of FDC products containing only NSAIDs. ${ }^{1}$ There is no synergistic effect with these combinations since they are targeting the same enzyme, and there is no rational reason to use such products. Patients not only are paying more, but are putting themselves at risks for adverse events by using NSAID FDCs. ${ }^{1}$ FDC's with two NSAIDs is not recommended due to the additive gastrointestinal risks. ${ }^{3}$ Other hazardous combinations available in India include antipsychotic FDC's, such as chlorpromazine, trifluoperazine, and trihexyphenidyl. These combination contains two antipsychotics associated with significant CNS toxicity and cardiotoxicity (sudden death). ${ }^{3}$

Naturally there are some good FDC's marketed in India, including antiparkinson agent carbidopa and levodopa, antitubercular, and antiretrovirals, but unfortunately majority of FDC's available in India fall in the "irrational" category.

FDCs are widely used in India; and there are more unapproved products than approved on the market. ${ }^{3}$ Unapproved formulations are commonly sold, even ones that are restricted, banned, or never approved in other countries, besides India, due to significant adverse events, including death. ${ }^{3}$

As PharmD students from the United States doing our advanced pharmacy practice rotation in India, availability of these "irrational" combinations struck us as the biggest challenge for "pharmacy practice" in India. The major goal of a clinical pharmacist is to improve patient outcome while reducing healthcare expenditures. In most cases, these FDCs will produce an opposite outcome. If the pharmacy profession has to gain strength and grow in India, this epidemic of "irrational medications" has to be addressed immediately.

\section{CONFLICT OF INTEREST}

The authors declare no conflict of interest.

\section{ABBREVIATIONS}

FDC: Fixed Dose Combination; CDSCO: Central Drug Standards Control Organization; NSAID: Non-Steroidal Anti-Inflammatory Drugs; CNS: Central Nervous System.

\section{REFERENCES}

1. Gautam CS, Saha L. Fixed dose drug combinations (FDCs): Rational or irrational: a view point. British journal of clinical pharmacology. 2008;65(5):795-6.

2. Gupta YK, Ramachandran SS. Fixed dose drug combinations: Issues and challenges in India. Indian journal of pharmacology. 2016;48(4):347.

3. McGettigan P, Roderick P, Mahajan R, Kadam A, Pollock AM. Use of fixed dose combination (FDC) drugs in India: central regulatory approval and sales of FDCs containing non-steroidal anti-inflammatory drugs (NSAIDs), metformin, or psychotropic drugs. PLoS medicine. 2015;12(5):e1001826.

4. Revikumar KG, Veena R. Fixed Dose Combinations in Indian Medicine Market-A SWOT Analysis. MOJ Bioequiv Availab. 2017;4(3):00071. 